\title{
Gewichtsabnahme bei Vorhofflimmern lohnt sich
}

Übergewichtige und adipöse Personen haben ein verdoppeltes Risiko für die
Entwicklung von Vorhofflimmern. Unklar ist, welche Auswirkungen eine lang-
fristige Gewichtsreduktion auf kardiale Parameter und den Herzrhythmus hat.

n Australien wurden 355 Frauen und Männer im Alter von durchschnittlich 63 Jahren, die unter paroxysmalem und chronischem Vorhofflimmern, nicht aber an anderen Herz-Kreislauf-Krankheiten litten, einer Gewichtsreduktion unterzogen. Sie standen unter Sotalol oder Flecainid. Ziel war, das Gewicht um $10 \mathrm{~kg}$ zu vermindern, was durch eine Umstellung der Ernährung (proteinreich, niedriger glykämischer Index, energiereduziert) und vermehrte Bewegung (Steigerung bis auf 200 Minuten/Woche) erreicht werden sollte. Die Gewichtsabnahme wurde in drei Gruppen beurteilt: $>10 \mathrm{~kg}, \quad 3-9 \mathrm{~kg}$, $<3 \mathrm{~kg}$. Die Beobachtung dauerte 48 , 46 bzw. 48 Monate.

Patienten mit einer Gewichtsabnahme von über $10 \mathrm{~kg}$ hatten eine sechsfach größere Chance, einen Sinusrhythmus zu erreichen, als die Patienten aus den anderen beiden Gruppen. Wer mehr als $5 \mathrm{~kg}$ abnahm, dessen Vorhofflimmern verschwand doppelt so häufig wie bei Personen ohne Gewichtsabnahme.

Am Ende der Beobachtung waren in Gruppe $145 \%$, in Gruppe $222 \%$ und in Gruppe $313 \%$ der Probanden frei von Vorhofflimmern ohne antiarrhythmische Pharmaka und ohne Ablation. Wer zwischenzeitlich wieder an Gewicht zunahm („weight fluctuation“), hatte nur einen halb so großen positiven Effekt.

Je stärker ausgeprägt die Gewichtsabnahme war, desto deutlicher besserten sich auch kardiovaskuläre Risikofaktoren wie systolischer Blutdruck, PlasmaInsulin, LDL-Cholesterin, Triglyzeride und hsCRP. Ebenso nahmen das Volumen des linken Vorhofs, die Dicke des interventrikularen Septums, der linksventrikuläre enddiastolische Durchmesser und das E/E'-Verhältnis ab.

\section{Kommentar}

In Anbetracht der Tatsache, dass mehr als die Hälfte der deutschen Erwachsenen aufgrund ihres erhöhten Body-Mass-Indexes (BMI) ein gesteigertes Risiko für die Entwicklung von Vorhofflimmern haben, ist die Frage, ob diese Krankheit durch eine Gewichtsreduktion reversibel ist, von groBem klinischem Interesse.

Experimentelle Studien in den letzten Jahren zeigten, dass eine Gewichtsabnahme die Häufigkeit von Vorhofflimmern reduziert. Unklar war bisher, ob diese Effekte auch von längerer Dauer sind und ob eine Schwelle hinsichtlich der Gewichtsabnahme besteht.

Weshalb ist Vorhofflimmern bei Gewichtsreduktion in Abhängigkeit des Ausmaßes der Gewichtsreduzierung reversibel? Adipositas erhöht den Blutdruck und das Blutvolumen, der Sympathikus ist aktiviert. Eine Gewichtsabnahme führt zu einer Druck- und Volumenreduktion mit verminVorhof verkleinert, die Wanddicken im Vorhof und Ventrikel vermindert und damit die linksventrikuläre Hypertrophie reduziert (kardiales Remodeling). Auch die oben erwähnten metabolischen kardiovaskulären Risikofaktoren werden vermindert; sie sind allesamt Risikofaktoren für die Entstehung von Vorhofflimmern.

Inzwischen liegen genügend valide Daten vor, um übergewichtigen und adipösen Patienten mit Vorhofflimmern eine Gewichtsreduktion zu empfehlen, wenn die antiarrhytmische Therapie nicht zum Erfolg geführt hat.

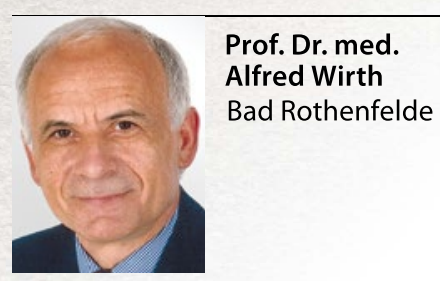
dertem sympathischem Antrieb, was den 